\title{
Concentration-dependent polymorphism of insulin amyloid fibrils
}

\author{
Andrius Sakalauskas ${ }^{1}$, Mantas Ziaunys ${ }^{1}$, Vytautas Smirnovas ${ }^{\text {Corresp. } 1}$ \\ ${ }^{1}$ Department of Biothermodynamics and Drug Design, Vilnius University, Life Sciences Center, Institute of Biotechnology, Vilnius, Lithuania \\ Corresponding Author: Vytautas Smirnovas \\ Email address: vytautas@smirnovas.info
}

Protein aggregation into highly structured fibrils has long been associated with several neurodegenerative disorders, such as Alzheimer's or Parkinson's disease. Polymorphism of amyloid fibrils increases the complexity of disease mechanisms and may be one of the reasons for the slow progress in drug research. Here we report protein concentration as another factor leading to polymorphism of insulin amyloid fibrils. Moreover, our data suggests that insulin amyloid conformation can self-replicate only via elongation, while seed-induced nucleation will lead to environment-defined conformation of fibrils. As similar observations were already described for a couple of other amyloid proteins, we suggest it to be a generic mechanism for self-replication of different amyloid fibril conformations. 
1 Concentration-dependent polymorphism of insulin amyloid

2 fibrils

3

4

5

\author{
Andrius Sakalauskas, Mantas Ziaunys, Vytautas Smirnovas \\ Institute of Biotechnology, Life Sciences Center, Vilnius University, Vilnius, Lithuania \\ Corresponding Author: \\ Vytautas Smirnovas \\ Sauletekio al. 7, Vilnius, LT-10257, Lithuania \\ Email address: vytautas.smirnovas@bti.vu.lt
}

\begin{abstract}
Protein aggregation into highly structured fibrils has long been associated with several neurodegenerative disorders, such as Alzheimer's or Parkinson's disease. Polymorphism of amyloid fibrils increases the complexity of disease mechanisms and may be one of the reasons for the slow progress in drug research. Here we report protein concentration as another factor leading to polymorphism of insulin amyloid fibrils. Moreover, our data suggests that insulin amyloid conformation can self-replicate only via elongation, while seed-induced nucleation will lead to environment-defined conformation of fibrils. As similar observations were already described for a couple of other amyloid proteins, we suggest it to be a generic mechanism for self-replication of different amyloid fibril conformations.
\end{abstract}

\title{
Introduction
}

Protein aggregation into amyloid fibrils has been linked to multiple neurodegenerative disorders, including Alzheimer's, Parkinson's and infectious prion diseases (Chiti and Dobson 2017; Knowles, Vendruscolo, and Dobson 2014), which affect tens of millions of people worldwide and is predicted to become even more prominent as the average human lifespan continues to increase (Isik 2010). Matters are further complicated by the fact that very few drugs have reached stage four of clinical trials and no efficient treatment or cure is available (Cummings et al. 2019; Mehta et al. 2017). One of the main reasons for such limited progress in the development of potential cures may be the complexity of fibril formation mechanisms (Meisl et al. 2017; Doig et al. 2017; Nasica-Labouze et al. 2017), as well as polymorphism of amyloid aggregates (Stein and True 2014).

The ability of the same protein to adopt distinct pathogenic conformations was first reported in studies of infectious prions and such conformations were referred to as strains (Safar et al. 1998; 
37 Collinge and Clarke 2007). Recently strain-like polymorphism was reported for a number of amyloid proteinsin vivo (Lu et al. 2013; Watts et al. 2014; Fändrich et al. 2018; Yamasaki et al. 2019), in vitro (Heise et al. 2005; Paravastu et al. 2008; Debelouchina et al. 2010; Dinkel et al. 2011; Bousset et al. 2013), and in silico (Pellarin et al. 2010). A number of environmental factors including pH (Sneideris et al. 2015), temperature (Tanaka et al. 2006; Colby et al. 2009), concentration of co-solvents (Dzwolak et al. 2004; Chatani et al. 2012), denaturants (Colby et al. 2009; Cobb et al. 2014) or salts (Morel et al. 2010; Bousset et al. 2013), as well as agitation (Petkova et al. 2005; Ostapchenko et al. 2010) can lead to different conformations of amyloid fibrils. Enormous amounts of data must be collected and analyzed in order to understand the complex effects of the environment on polymorphism of amyloids.

Due to its relatively low cost, wide availability and simple aggregation protocols, insulin became one of the most common proteins used to study amyloid fibril formation. Several years ago, we summarized available data on polymorphism of insulin amyloid fibrils and came with the hypothesis that the number of insulin amyloid conformations may be limited to two and the major factor which determines formation of different strains is a shift of the equilibrium between insulin monomers and dimers (oligomers) (Sneideris et al. 2015). Our current data supports the existence of a third conformation of insulin amyloid fibrils and suggests that polymorphism of insulin amyloid fibrils is more complex.

\section{Materials and Methods}

\section{Insulin sample preparation}

Human recombinant insulin powder (Sigma-Aldrich cat. No. 91077C) was dissolved in a 20\% acetic acid solution containing $100 \mathrm{mM} \mathrm{NaCl}$ (reaction solution) to a final concentration of 2 $\mathrm{mM}(11.6 \mathrm{mg} / \mathrm{ml})$. Insulin concentration was determined by measuring the sample's absorbance at $280 \mathrm{~nm} \varepsilon=6335 \mathrm{M}^{-1} \mathrm{~cm}^{-1}, \mathrm{M}=5808 \mathrm{Da}$. Samples for unseeded aggregation kinetic measurements were prepared by diluting the $2 \mathrm{mM}$ stock solution using the reaction solution and $10 \mathrm{mM}$ ThT stock solution to a range of concentrations from $0.2 \mathrm{mM}$ to $1.0 \mathrm{mM}$ (which contained $100 \mu \mathrm{M}$ of ThT). For seeded aggregation, insulin fibrils prepared from the $0.2 \mathrm{mM}$ and $1.0 \mathrm{mM}$ samples were sonicated for $10 \mathrm{~min}$ using Sonopuls 3100 (Bandelin) ultrasonic homogenizer equipped with a MS73 tip (40\% power, 30 s sonication/ $30 \mathrm{~s}$ rest intervals). The homogenized fibrils were then diluted with the reaction solution and mixed with the $2 \mathrm{mM}$ insulin and $10 \mathrm{mM}$ ThT stock solutions to yield $0.2 \mathrm{mM}$ and $1.0 \mathrm{mM}$ concentration samples containing $100 \mu \mathrm{M}$ ThT and a range of fibril concentrations (from $5 \%$ to $10^{-6} \%$ of monomer mass).

\section{Aggregation kinetics}

Insulin aggregation kinetics were monitored at $60{ }^{\circ} \mathrm{C}$ without agitation by measuring ThT fluorescence emission intensity (excitation wavelength - $440 \mathrm{~nm}$, emission - $480 \mathrm{~nm}$ ) through the bottom of a 96 well non-binding surface plate using Synergy H4 Hybrid Multi-Mode (Biotek) 
74 plate reader (readouts were taken every $10 \mathrm{~min}$ to minimize plate agitation). For every condition

4 independent measurements were performed. Aggregation half-times $\left(t_{50}\right)$ were calculated as the time needed to reach $50 \%$ of signal intensity. The full concentration range of aggregated insulin samples were centrifuged at $10000 \mathrm{~g}$ for $30 \mathrm{~min}$ and the residual unaggregated insulin in the supernatant was determined to be less than $1 \%$ of initial protein concentration.

\section{Atomic Force Microscopy (AFM)}

After kinetic measurements, samples were diluted with the reaction solution to a $50 \mu \mathrm{M}$ concentration and $20 \mu \mathrm{L}$ of each was deposited on freshly cleaved mica and incubated for $1 \mathrm{~min}$. Subsequently, samples were rinsed with $1 \mathrm{~mL}$ of MilliQ water and dried under gentle airflow. Three-dimensional AFM maps were acquired using a Dimension Icon (Bruker) atomic force microscope operating in tapping mode and equipped with a silicon cantilever Tap300AI-G (40 N $\mathrm{m}^{-1}$, Budget Sensors) with a typical tip radius of curvature of $8 \mathrm{~nm}$. High-resolution (1024 x 1024 pixels) images were acquired. The scan rate was $1 \mathrm{~Hz}$. AFM images were flattened and analyzed using SPIP (Image Metrology).

\section{Fourier-Transform Infrared (FTIR) Spectroscopy}

Insulin fibrils were separated from solution by centrifugation at $10000 \mathrm{~g}$ for $30 \mathrm{~min}$ and subsequently resuspended in $1 \mathrm{~mL}$ of $\mathrm{D}_{2} \mathrm{O}$, the procedure was repeated three times. Then the fibrils were resuspended in $0.2 \mathrm{~mL}$ of $\mathrm{D}_{2} \mathrm{O}$ and sonicated for 1 min using a MS72 tip (with 20\% power and constant sonication). Samples were deposited between two $\mathrm{CaF}_{2}$ transmission windows separated by $0.05 \mathrm{~mm}$ teflon spacers. The FTIR spectra were recorded using Vertex 80v (Bruker) IR spectrometer equipped with a mercury cadmium telluride detector, at room temperature under vacuum ( $\sim 2 \mathrm{mBar})$ conditions. 256 interferograms of $2 \mathrm{~cm}^{-1}$ resolution were averaged for each spectrum. Spectrum of $\mathrm{D}_{2} \mathrm{O}$ was subtracted from the spectrum of each sample. All spectra were normalized to the same area of amide I/I' band (1700-1595 $\left.\mathrm{cm}^{-1}\right)$. All data processing was performed using GRAMS software.

\section{Results}

\section{Fibril formation at different concentrations}

Aggregating a range of insulin concentrations in $20 \%$ acetic acid with $100 \mathrm{mM} \mathrm{NaCl}$ at $60{ }^{\circ} \mathrm{C}$ without agitation reveals a typical kinetic curve pattern, where an increasing insulin concentration leads to shorter aggregation times (Fig. 1A). However, we observe an uneven ratio distribution between ThT fluorescence emission intensities and final fibril concentrations (Fig. 1B). As the concentration of insulin in the sample increases, this ratio shifts ten-fold, indicating either a higher quantum yield or considerably more bound ThT molecules. 
114 The FTIR spectra of aggregated samples exhibit maxima in the amide I/I' region at $\sim 1628 \mathrm{~cm}^{-1}$

115

116

117

118

119

120

121

122

123

124

125

126

127

128

129

130

131

132

133

134

135

136

137

138

139

140

141

142

143

144

145

146

147

148

149

150

151

152

153 with the shoulder at $\sim 1641 \mathrm{~cm}^{-1}$, and a small band outside of the amide I/I' region at $\sim 1729 \mathrm{~cm}^{-1}$ (Fig. 1C), which is very similar to previously reported insulin fibrils formed in phosphate buffer at $\mathrm{pH} \leq 2$ [Sneideris2015]. However, minor concentration-dependent differences can be observed (Fig. 1C and D). The spectra of fibrils, formed at lower insulin concentrations, have a pronounced shoulder at $1641 \mathrm{~cm}^{-1}$, while a minor band at $1620 \mathrm{~cm}^{-1}$ appears in the second derivative spectra (Fig. 1D) of samples aggregated at higher protein concentrations. The $1.0 \mathrm{mM}$ and $0.8 \mathrm{mM}$ fibril spectra are nearly identical, while $0.6 \mathrm{mM}$ and $0.4 \mathrm{mM}$ spectra appear to be intermediates between $0.8 \mathrm{mM}$ and $0.2 \mathrm{mM}$, suggesting the existence of two distinct conformations.

\section{Fibril morphology}

The morphology of insulin fibrils formed at different concentrations was compared using AFM. We can see far more small and separated aggregates in samples formed at lower insulin concentration (Fig. 2A-E). Analysis of variance (ANOVA) reveals that there is a statistically relevant fibril height difference $(p=0.01, n=50)$ between the low and high concentration samples (Fig. 2F). Additional AFM images of these conditions are available as supplementary information (Supplementary Fig. S1).

\section{Seeded aggregation}

In order to determine whether observed different fibril templates can propagate at unfavorable conditions, a set of seeded aggregation reactions were performed (Fig. 3A-D, Supplementary Fig. S2). In all four cases we observe a fibril-concentration-dependent seeding propensity (Fig. $3 \mathrm{E})$, however, there is an interesting ThT fluorescence distribution, based on the amount and type of seed added (Fig. 3F). When the low concentration fibrils $(0.2 \mathrm{mg} / \mathrm{ml}$, further on referred as LCF) are added to $0.2 \mathrm{mM}$ insulin solutions, there are relatively no major differences in the fluorescence intensity at the end of each reaction. The same can be said in the case when the high concentration fibrils $(1.0 \mathrm{mg} / \mathrm{ml}$, further on referred as HCF) are added to $1.0 \mathrm{mM}$ insulin solutions. However, when the LCF are added to $1.0 \mathrm{mM}$ insulin solutions, high seed concentrations yield a low fluorescence intensity, which then rises with decreased amount of seeds, eventually resulting in an intensity comparable to HCF. The opposite is observed when $\mathrm{HCF}$ are added to $0.2 \mathrm{mM}$ insulin solutions, where high initial fibril concentrations yield an intensity comparable to the seed conformation (when accounted for fibril concentration at the end of the reaction) and an intensity similar to LCF when the seed concentration becomes minimal.

In order to further confirm the self-replication ability of both conformations, fibrils formed during seeded aggregation were examined by FTIR and their spectra were compared to the unseeded aggregation fibril spectra (Fig. 4A-D). The results show that when a large concentration of preformed fibrils is added to either $0.2 \mathrm{mM}$ or $1.0 \mathrm{mM}$ insulin solutions, the seed self-replicates and maintains its initial secondary structure. On the other hand, when a low 
154 concentration of seed is added, the resulting FTIR spectra are similar to their respective

155 environment conformations, rather than the seed.

156 Seeded fibril morphology

157 When large amounts of sonicated aggregates are used, there is minimal difference in the length

158 and distribution of fibrils (Fig. 5A-D), likely due to the large amount of aggregation centers.

159 When the amount of seed used is low, the fibril length and distribution is similar to unseeded

160 aggregation (Fig. 5E-H). Fibril height distribution reveals a similarity between almost all

161 conditions, except for when $1.0 \mathrm{mM}$ insulin is seeded with low concentrations of either

162 conformation (Fig. 5I), where the height distribution is comparable to unseeded nucleation.

163 Additional AFM images of these conditions are available as supplementary information

164 (Supplementary Fig. S3).

165 Discussion

166 The first and most apparent difference between the samples, aggregated at different protein concentrations is their ability to enhance ThT fluorescence. A very similar effect was reported in case of protein-concentration-dependent polymorphism of glucagon amyloid fibrils (Pedersen et al. 2006). A 10-fold increase in ThT binding positions is highly improbable and slightly different fibril size distribution seen in AFM images could not strongly affect the number of binding positions, so a more appropriate explanation could be changes in the fibril's surface, facilitating a different ThT binding mode, as insulin fibrils have been shown to possess more than one way of incorporating ThT molecules (Groenning et al. 2007). Similar differences in ThT fluorescence were observed with different conformations of alpha-synuclein fibrils and attributed to the different binding of ThT molecules (Sidhu et al. 2018), so we can hypothesize that a low protein concentration leads to a different conformation of insulin amyloid fibrils.

Protein-concentration-dependent polymorphism of insulin amyloid fibrils is supported also by different FTIR spectra. In fact, spectral differences are rather minor in comparison to the ones observed between spectra of previously reported insulin conformations (Dzwolak et al. 2004; Sneideris et al. 2015), but the hallmark of each spectrum is conserved in seeding experiments (Fig. 4A and B), which supports the hypothesis of different amyloid conformations. Comparison of FTIR spectra to the previously reported data (Sneideris et al. 2015) suggests that the fibril conformation formed at higher insulin concentrations is the same as previously reported, while the one formed at lower concentrations falls out of the previously proposed scheme (Sneideris et 185 al. 2015).

Atomic force microscopy data does not add much of information. It seems that average size of spontaneously formed fibrils slightly increases with higher protein concentration (Fig. 2), but this effect does not depend on the type of seeds (Fig. 5).

Currently we are aware of two mechanisms of seed-induced aggregation. One is amyloid fibril 
192

193

194

195

196

197

198

199

200

201

202

203

204

205

206

207

208

209

210

211

212

213

214

215

216

217

218

219

220

221

222

223

224

225

226

227

228

secondary nucleation (Meisl et al. 2016; Fodera et al. 2008; Scheidt et al. 2019; Törnquist et al. 2018; Bunce et al. 2019)). Previously we have demonstrated that in case of cross-seeding of different environment-induced conformations of prion protein amyloid fibrils, the conformational template can self-propagate only via elongation mechanism, while surface induced nucleation only speeds up the aggregation process, but the conformation is defined by the environment conditions (Sneideris, Milto, and Smirnovas 2015). Recently similar observations were reported on amyloid beta (Brännström et al. 2018) and alpha synuclein (Peduzzo, Linse, and Buell 2019). Our cross-environment seeding data on insulin follows the same path. With higher amount of seeds, the aggregation kinetic curves are exponential, which means that the majority of the protein is aggregated via elongation of seeds - in such case the final relative ThT fluorescence intensity and FTIR spectra of seeds and final aggregates are very similar. Lowering the amount of seeds leads to sigmoid kinetic curves which means that the majority of the protein is aggregated via new-formed nuclei and seeds are mainly employed as catalyzers - in such case the final relative ThT fluorescence intensity and FTIR spectra of seeds and final aggregates are different.

\section{Conclusions}

Generally, in the seeded growth experiment of amyloid fibrils one expects self-replication of seed conformation. Here we showed that such expectations are valid only at certain circumstances - amyloid fibrils self-replicate their conformation only via elongation, else the conformation of aggregates is environment-dependent. As similar conclusions were previously derived in studies of prion protein, amyloid beta, and alpha-synuclein, there may be enough data to consider it as a general way for self-replication of different amyloid fibril conformations.

\section{Acknowledgments}

The authors acknowledge Prof. G. Niaura from the Center of Physical Sciences and Technology for the access to FTIR.

\section{References}

Bousset, Luc, Laura Pieri, Gemma Ruiz-Arlandis, Julia Gath, Poul Henning Jensen, Birgit Habenstein, Karine Madiona, Vincent Olieric, Anja Böckmann, Beat H. Meier, Ronald Melki. 2013. "Structural and Functional Characterization of Two Alpha-Synuclein Strains." Nature Communications 4. https://doi.org/10.1038/ncomms3575.

Brännström, Kristoffer, Tohidul Islam, Anna L. Gharibyan, Irina Iakovleva, Lina Nilsson, Cheng Choo Lee, Linda Sandblad, Annelie Pamrén, and Anders Olofsson. 2018. "The Properties of Amyloid- $\beta$ Fibrils Are Determined by Their Path of Formation." Journal of Molecular Biology 430 (13): 1940-49. https://doi.org/10.1016/j.jmb.2018.05.001. 
Bunce, Samuel J., Yiming Wang, Katie L. Stewart, Alison E. Ashcroft, Sheena E. Radford, Carol K. Hall, and Andrew J. Wilson. 2019. "Molecular Insights into the Surface-Catalyzed Secondary Nucleation of Amyloid- $\beta 40$ (A $\beta 40$ ) by the Peptide Fragment A $\beta$ 16-22." Science Advances 5 (6): eaav8216. doi:10.1126/sciadv.aav8216.

Chatani, Eri, Hisashi Yagi, Hironobu Naiki, and Yuji Goto. 2012. "Polymorphism of $\beta 2$ Microglobulin Amyloid Fibrils Manifested by Ultrasonication-Enhanced Fibril Formation in Trifluoroethanol." Journal of Biological Chemistry 287 (27): 22827-37. https://doi.org/10.1074/jbc.M111.333310.

Chiti, Fabrizio, and Christopher M. Dobson. 2017. "Protein Misfolding, Amyloid Formation, and Human Disease: A Summary of Progress Over the Last Decade." Annual Review of Biochemistry 86 (1): 27-68. https://doi.org/10.1146/annurev-biochem-061516-045115.

Cobb, Nathan J., Marcin I. Apostol, Shugui Chen, Vytautas Smirnovas, and Witold K. Surewicz. 2014. "Conformational Stability of Mammalian Prion Protein Amyloid Fibrils Is Dictated by a Packing Polymorphism within the Core Region." Journal of Biological Chemistry 289 (5): 2643-50. https://doi.org/10.1016/j.jmb.2010.05.051.

Colby, David W., Kurt Giles, Giuseppe Legname, Holger Wille, Ilia V. Baskakov, Stephen J. DeArmond, and Stanley B. Prusiner. 2009. "Design and Construction of Diverse Mammalian Prion Strains." Proceedings of the National Academy of Sciences 106 (48): 20417-22. https://doi.org/10.1073/pnas.0910350106.

Collinge, John, and Anthony R. Clarke. 2007. "A General Model of Prion Strains and Their Pathogenicity.” Science 318 (5852): 930-36. https://doi.org/10.1126/science.1138718.

Cummings, Jeffrey, Garam Lee, Aaron Ritter, Marwan Sabbagh, and Kate Zhong. 2019. “Alzheimer's Disease Drug Development Pipeline: 2019." Alzheimer's \& Dementia: Translational Research \& Clinical Interventions 5: 272-93. https://doi.org/10.1016/j.trci.2019.05.008.

Debelouchina, Galia T., Geoffrey W. Platt, Marvin J. Bayro, Sheena E. Radford, and Robert G. Griffin. 2010. "Magic Angle Spinning NMR Analysis of $\beta 2$-Microglobulin Amyloid Fibrils in Two Distinct Morphologies." Journal of the American Chemical Society 132 (30): 10414-23. https://doi.org/10.1021/ja102775u.

Dinkel, Paul D., Ayisha Siddiqua, Huy Huynh, Monil Shah, and Martin Margittai. 2011. "Variations in Filament Conformation Dictate Seeding Barrier between Three- and FourRepeat Tau." Biochemistry 50 (20): 4330-36. https://doi.org/10.1021/bi2004685.

Doig, Andrew J., Maria P. del Castillo-Frias, Olivia Berthoumieu, Bogdan Tarus, Jessica NasicaLabouze, Fabio Sterpone, Phuong H. Nguyen, Nigel M. Hooper, Peter Faller, and Philippe Derreumaux. 2017. "Why Is Research on Amyloid- $\beta$ Failing to Give New Drugs for Alzheimer's Disease?” ACS Chemical Neuroscience 8 (7): 1435-37. https://doi.org/10.1021/acschemneuro.7b00188.Dzwolak, Wojciech, Vytautas Smirnovas, Ralf Jansen, and Roland Winter. 2004. "Insulin Forms Amyloid in a Strain-Dependent Manner: An FT-IR Spectroscopic Study.” Protein Science 13 (7): 1927-32. https://doi.org/10.1110/ps.03607204.

Fändrich, M., S. Nyström, K. P. R. Nilsson, A. Böckmann, H. LeVine, and P. Hammarström. 
2018. "Amyloid Fibril Polymorphism: A Challenge for Molecular Imaging and Therapy." Journal of Internal Medicine 283 (3): 218-37. https://doi.org/10.1111/joim.12732.

Foderà, Vito, Fabio Librizzi, Minna Groenning, Marco van de Weert, and Maurizio Leone. 2008. "Secondary Nucleation and Accessible Surface in Insulin Amyloid Fibril Formation." The Journal of Physical Chemistry B 112 (12): 3853-58. https://doi.org/10.1021/jp710131u.

Groenning, Minna, Mathias Norrman, James M. Flink, Marco van de Weert, Jens T. Bukrinsky, Gerd Schluckebier, and Sven Frokjaer. 2007. "Binding Mode of Thioflavin T in Insulin Amyloid Fibrils." Journal of Structural Biology 159 (3): 483-97. https://doi.org/10.1016/j.jsb.2007.06.004.

Heise, Henrike, Wolfgang Hoyer, Stefan Becker, Ovidiu C. Andronesi, Dietmar Riedel, and Marc Baldus. 2005. "Molecular-Level Secondary Structure, Polymorphism, and Dynamics of Full-Length -Synuclein Fibrils Studied by Solid-State NMR." Proceedings of the National Academy of Sciences 102 (44): 15871-76. https://doi.org/10.1073/pnas.0506109102.

Isik, Ahmet Turan. 2010. "Late Onset Alzheimer's Disease in Older People." Clinical Interventions in Aging 5 (October): 307-11. https://doi.org/10.2147/CIA.S11718.

Knowles, Tuomas P J, Michele Vendruscolo, and Christopher M. Dobson. 2014. "The Amyloid State and Its Association with Protein Misfolding Diseases." Nature Reviews Molecular Cell Biology 15 (6): 384-96. https://doi.org/10.1038/nrm3810.

Lu, Jun-Xia, Wei Qiang, Wai-Ming Yau, Charles D. Schwieters, Stephen C. Meredith, and Robert Tycko. 2013. "Molecular Structure of $\beta$-Amyloid Fibrils in Alzheimer's Disease Brain Tissue." Cell 154 (6): 1257-68. https://doi.org/10.1016/j.cell.2013.08.035.

Mehta, Dev, Robert Jackson, Gaurav Paul, Jiong Shi, and Marwan Sabbagh. 2017. "Why Do Trials for Alzheimer's Disease Drugs Keep Failing? A Discontinued Drug Perspective for 2010-2015." Expert Opinion on Investigational Drugs 26 (6): 735-39. https://doi.org/10.1080/13543784.2017.1323868.

Meisl, Georg, Julius B Kirkegaard, Paolo Arosio, Thomas C T Michaels, Michele Vendruscolo, Christopher M Dobson, Sara Linse, and Tuomas P J Knowles. 2016. "Molecular Mechanisms of Protein Aggregation from Global Fitting of Kinetic Models." Nature Protocols 11 (2): 252-72. https://doi.org/10.1038/nprot.2016.010.

Meisl, Georg, Luke Rajah, Samuel A. I. Cohen, Manuela Pfammatter, Anđela Šarić, Erik Hellstrand, Alexander K. Buell, Adriano Aguzzi, Sara Linse, Michele Vendruscolo, Christopher M. Dobson, Tuomas P. J. Knowles. 2017. "Scaling Behaviour and RateDetermining Steps in Filamentous Self-Assembly." Chemical Science 8 (10): 7087-97. https://doi.org/10.1039/C7SC01965C.

Morel, Bertrand, Lorena Varela, Ana I. Azuaga, and Francisco Conejero-Lara. 2010. "Environmental Conditions Affect the Kinetics of Nucleation of Amyloid Fibrils and Determine Their Morphology." Biophysical Journal 99 (11): 3801-10. https://doi.org/10.1016/j.bpj.2010.10.039.

Nasica-Labouze, Jessica, Phuong H. Nguyen, Fabio Sterpone, Olivia Berthoumieu, Nicolae- 
Viorel Buchete, Sébastien Coté, Alfonso De Simone, Andrew J. Doig, Peter Faller, Angel Garcia, Alessandro Laio, Mai Suan Li, Simone Melchionna, Normand Mousseau, Yuguang $\mathrm{Mu}$, Anant Paravastu, Samuela Pasquali, David J. Rosenman, Birgit Strodel, Bogdan Tarus, John H. Viles, Tong Zhang, Chunyu Wang, and Philippe Derreumaux. 2015. "Amyloid $\beta$ Protein and Alzheimer's Disease: When Computer Simulations Complement Experimental Studies." Chemical Reviews 115 (9): 3518-63. https://doi.org/10.1021/cr500638n.

Ostapchenko, Valeriy G., Michael R. Sawaya, Natallia Makarava, Regina Savtchenko, K. Peter R. Nilsson, David Eisenberg, and Ilia V. Baskakov. 2010. "Two Amyloid States of the Prion Protein Display Significantly Different Folding Patterns.” Journal of Molecular Biology 400 (4): 908-21. https://doi.org/10.1016/j.jmb.2010.05.051.

Paravastu, Anant K., Richard D. Leapman, W.-M. Yau, and Robert Tycko. 2008. "Molecular Structural Basis for Polymorphism in Alzheimer's -Amyloid Fibrils." Proceedings of the National Academy of Sciences 105 (47): 18349-54. https://doi.org/10.1073/pnas.0806270105.

Pedersen, Jesper Søndergaard, Dantcho Dikov, James L. Flink, Hans Aage Hjuler, Gunna Christiansen, and Daniel Erik Otzen. 2006. "The Changing Face of Glucagon Fibrillation: Structural Polymorphism and Conformational Imprinting." Journal of Molecular Biology 355 (3): 501-23. https://doi.org/10.1016/j.jmb.2005.09.100.

Peduzzo, Alessia, Sara Linse, and Alexander Buell. 2019. “The Properties of $\alpha$-Synuclein Secondary Nuclei Are Dominated by the Solution Conditions Rather than the Seed Fibril Strain." ChemRxiv. doi:10.26434/chemrxiv.9757778.v1.

Pellarin, Riccardo, Philipp Schuetz, Enrico Guarnera, and Amedeo Caflisch. 2010. “Amyloid Fibril Polymorphism Is under Kinetic Control." Journal of the American Chemical Society 132 (42): 14960-70. doi:10.1021/ja106044u.

Petkova, Aneta T., Richard D Leapman, Zhihong Guo, Wai-Ming Yau, Mark P Mattson, and Robert Tycko. 2005. "Self-Propagating, Molecular-Level Polymorphism in Alzheimer's Beta-Amyloid Fibrils." Science 307 (5707): 262-65. https://doi.org/10.1126/science.1105850.

Safar, Jiri, Holger Wille, Vincenza Itri, Darlene Groth, Hana Serban, Marilyn Torchia, Fred E. Cohen, and Stanley B. Prusiner. 1998. "Eight Prion Strains Have PrPSc Molecules with Different Conformations.” Nature Medicine 4 (10): 1157-65. https://doi.org/10.1038/2654.

Scheidt, Tom, Urszula Łapińska, Janet R. Kumita, Daniel R. Whiten, David Klenerman, Mark R. Wilson, Samuel I. A. Cohen, Sara Linse, Michele Vendruscolo, Christopher M. Dobson, Tuomas P. J. Knowles, and Paolo Arosio. 2019. "Secondary Nucleation and Elongation Occur at Different Sites on Alzheimer's Amyloid- $\beta$ Aggregates." Science Advances 5 (4): eaau3112. https://doi.org/10.1126/sciadv.aau3112.

Sidhu, Arshdeep, Jonathan Vaneyck, Christian Blum, Ine Segers-Nolten, and Vinod Subramaniam. 2018. "Polymorph-Specific Distribution of Binding Sites Determines Thioflavin-T Fluorescence Intensity in $\alpha$-Synuclein Fibrils.” Amyloid 25 (3): 189-96. https://doi.org/10.1080/13506129.2018.1517736.

Sneideris, Tomas, Domantas Darguzis, Akvile Botyriute, Martynas Grigaliunas, Roland Winter, 
and Vytautas Smirnovas. 2015. "pH-Driven Polymorphism of Insulin Amyloid-Like Fibrils.” PLoS One 10 (8): e0136602. https://doi.org/10.1371/journal.pone.0136602.

Sneideris, Tomas, Katažyna Milto, and Vytautas Smirnovas. 2015. "Polymorphism of Amyloidlike Fibrils Can Be Defined by the Concentration of Seeds." PeerJ 3 (August): e1207. https://doi.org/10.7717/peerj.1207.

Stein, Kevin C., and Heather L. True. 2014. "Prion Strains and Amyloid Polymorphism Influence Phenotypic Variation.” PLoS Pathogens 10 (9): e1004328. https://doi.org/10.1371/journal.ppat.1004328.

Tanaka, Motomasa, Sean R. Collins, Brandon H. Toyama, and Jonathan S. Weissman. 2006. "The Physical Basis of How Prion Conformations Determine Strain Phenotypes." Nature 442 (7102): 585-89. https://doi.org/10.1038/nature04922.

Törnquist, Mattias, Thomas C. T. Michaels, Kalyani Sanagavarapu, Xiaoting Yang, Georg Meisl, Samuel I. A. Cohen, Tuomas P. J. Knowles, and Sara Linse. 2018. "Secondary Nucleation in Amyloid Formation." Chemical Communications 54 (63): 8667-84. https://doi.org/10.1039/C8CC02204F.

Watts, Joel C., Carlo Condello, J. Stohr, Abby Oehler, Joanne Lee, Stephen J. DeArmond, Lars Lannfelt, Martin Ingelsson, Kurt Giles, and Stanley B. Prusiner. 2014. "Serial Propagation of Distinct Strains of A Prions from Alzheimer's Disease Patients." Proceedings of the National Academy of Sciences 111 (28): 10323-28. https://doi.org/10.1073/pnas.1408900111.

Yamasaki, Tritia R., Brandon B. Holmes, Jennifer L. Furman, Dhruva D. Dhavale, Bryant W. Su, Eun-Suk Song, Nigel J. Cairns, Paul T. Kotzbauer, and Marc I. Diamond. 2019. "Parkinson's Disease and Multiple System Atrophy Have Distinct $\alpha$-Synuclein Seed Characteristics." Journal of Biological Chemistry 294 (3): 1045-58. doi:10.1074/jbc.RA118.004471. 


\section{Figure 1}

Concentration-dependent differences of insulin aggregation.

Aggregation kinetics of unseeded insulin in $20 \%$ acetic acid with $100 \mathrm{mM} \mathrm{NaCl}$ at $60{ }^{\circ} \mathrm{C}$ without agitation followed by ThT fluorescence (A), insert shows aggregation kinetics of 0.2 $\mathrm{mM}$ insulin. Each kinetic data point is the average of 4 repeats. ThT fluorescence intensity and fibril concentration ratios (B). FTIR absorption (C) and second derivative (D) spectra of insulin fibrils. 

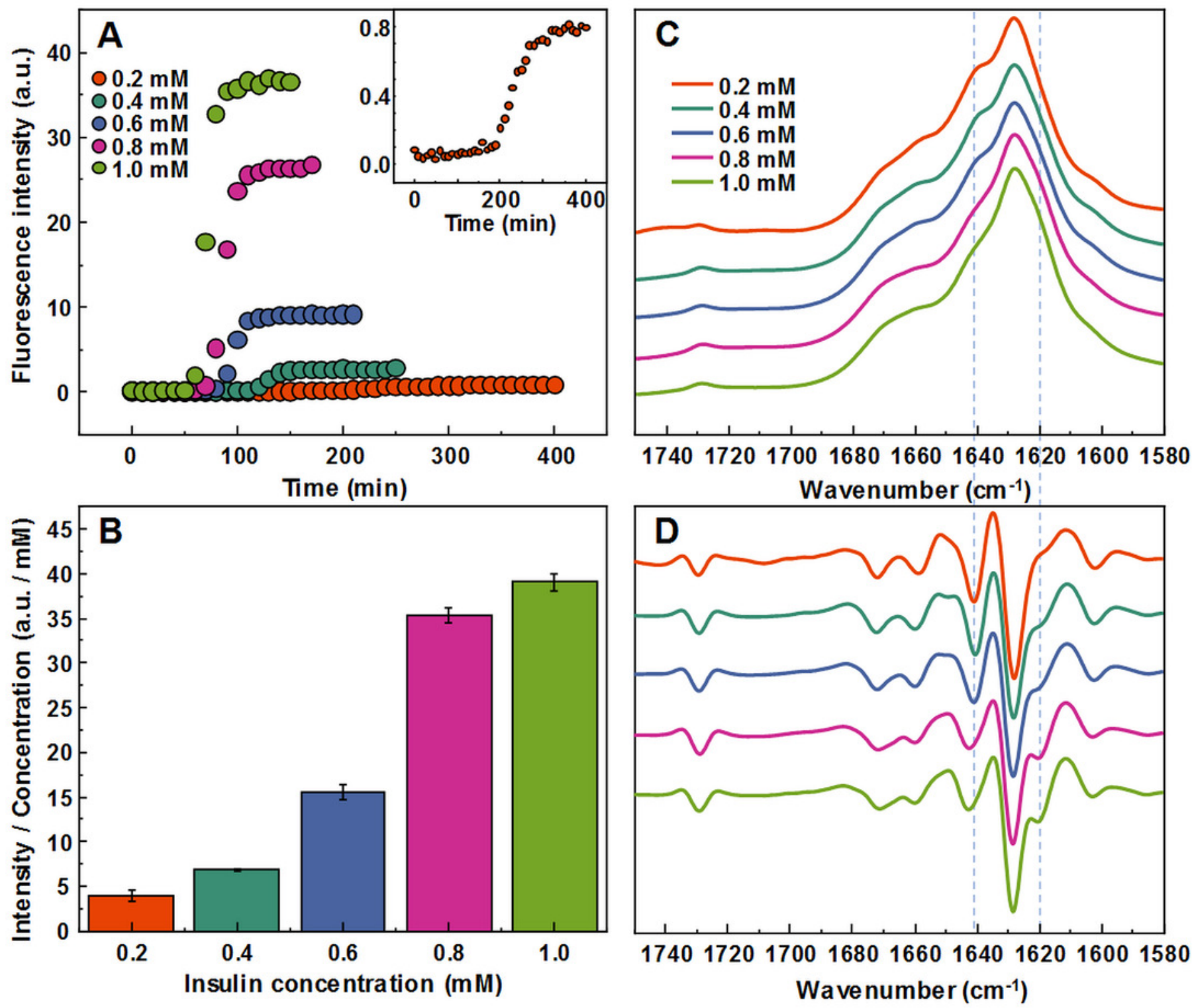


\section{Figure 2}

AFM analysis of insulin fibrils.

Insulin fibrils formed at $0.2 \mathrm{mM}(\mathrm{A}), 0.4 \mathrm{mM}(\mathrm{B}), 0.6 \mathrm{mM}(\mathrm{C}), 0.8 \mathrm{mM}(\mathrm{D})$ and $1.0 \mathrm{mM}(\mathrm{E})$ concentrations. Insulin fibril height distribution with box plots indicating the interquartile range and errors bars are for 1 standard deviation (F). Sample size for each ANOVA test was 50.
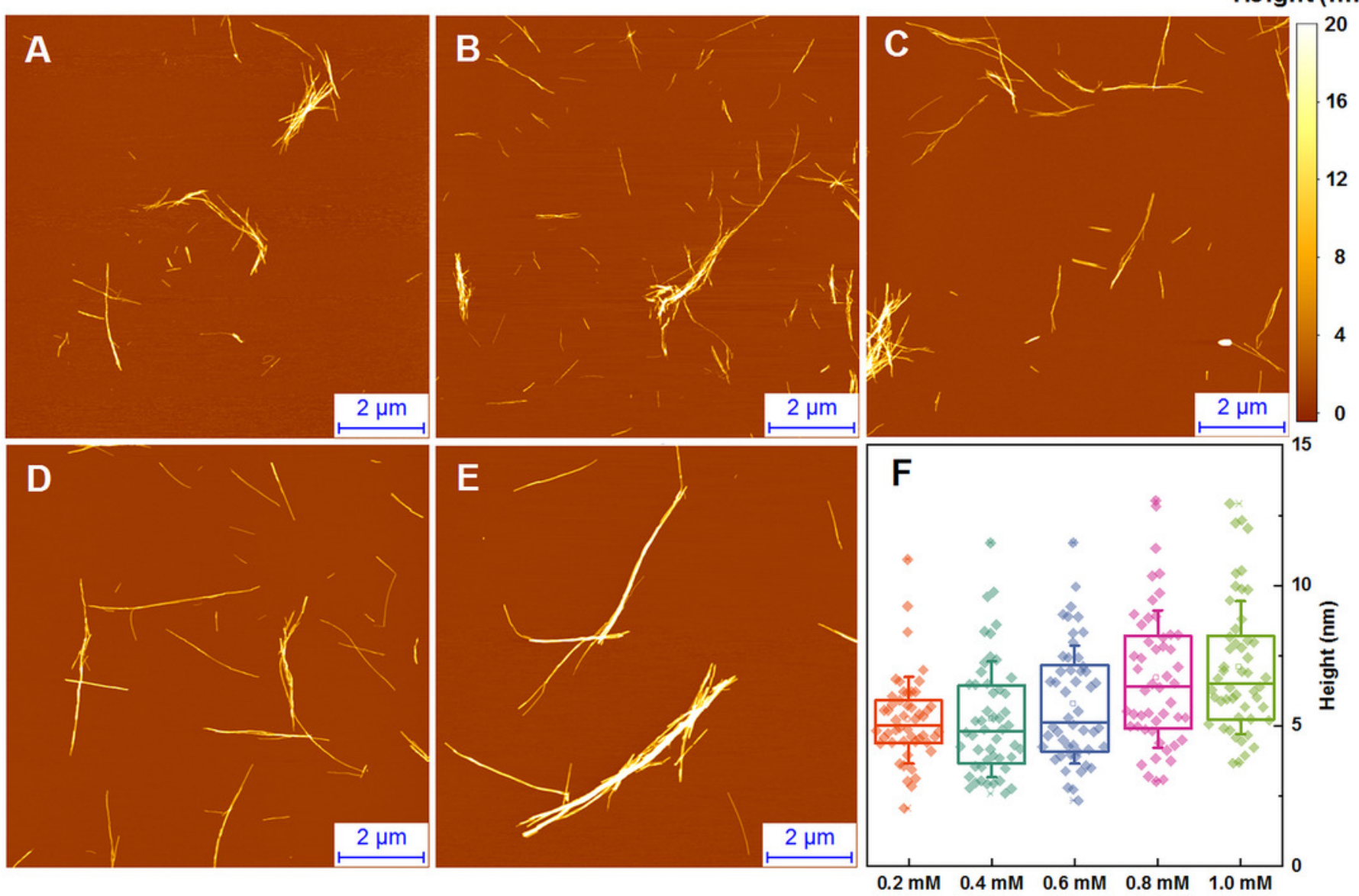


\section{Figure 3}

Seeded aggregation of insulin with a range of preformed fibrils.

Aggregation kinetics of insulin where the LCF are added to $0.2 \mathrm{mM}$ insulin solutions (A), LCF

to $1.0 \mathrm{mM}(B), H C F$ to $0.2 \mathrm{mM}(C)$ and HCF to $1.0 \mathrm{mM}(D)$. Aggregation half-time $\left(t_{50}\right)$

dependence on concentration and type of seed added (E). ThT fluorescence intensity and fibril concentration ratio dependence on added seed concentration (F). Each data point is the average of 4 repeats. 
$\bigcirc 5 \% \bigcirc 1 \% \bigcirc 10^{-4} \% \bigcirc 10^{-6} \% \bigcirc$ Control
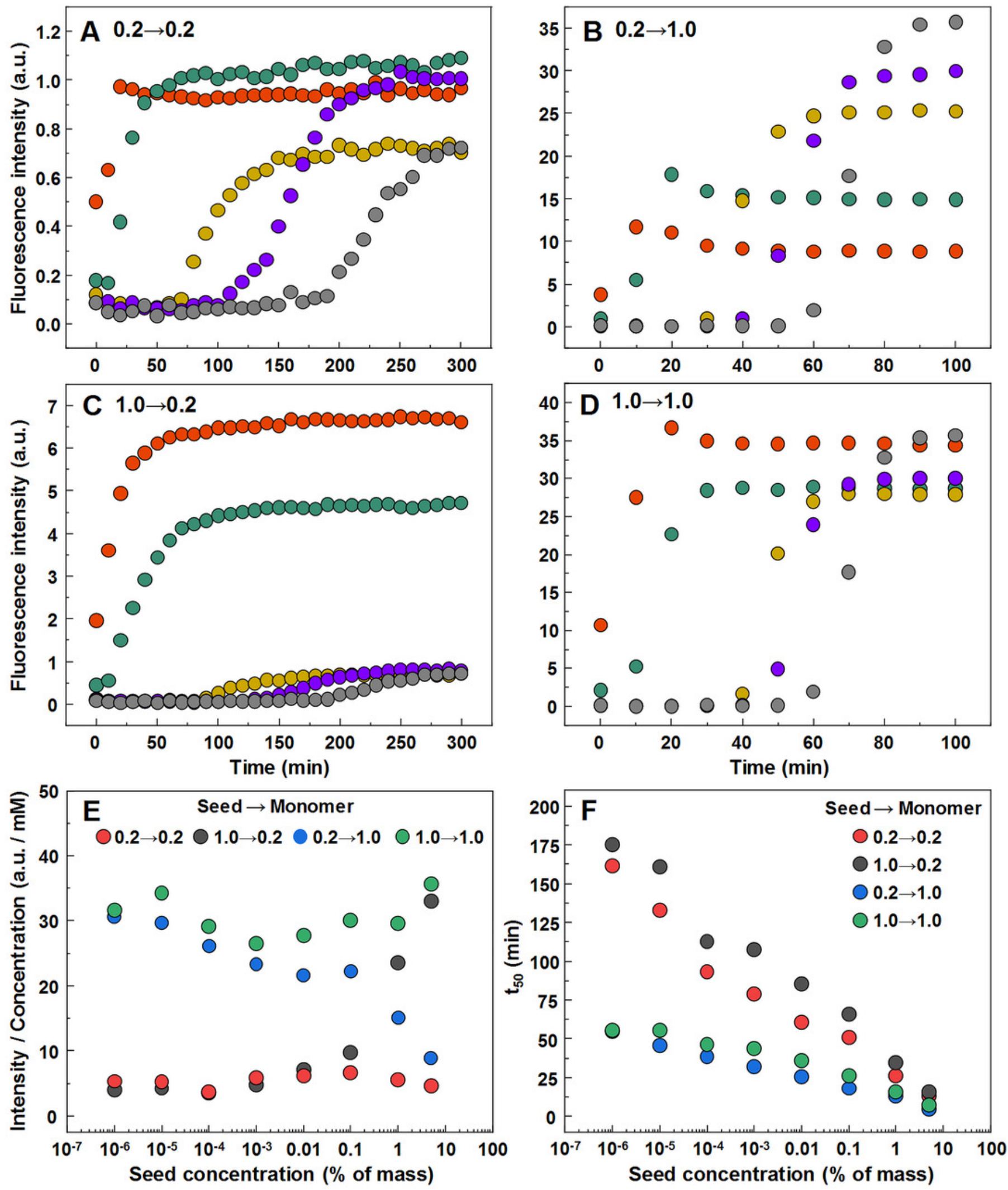
Figure 4

FTIR analysis of seeded aggregates.

Absorption and second derivate spectra of insulin fibrils when 5\% (A and B respectively) and $0.0001 \%$ ( $C$ and $D$ respectively) preformed fibrils are added.

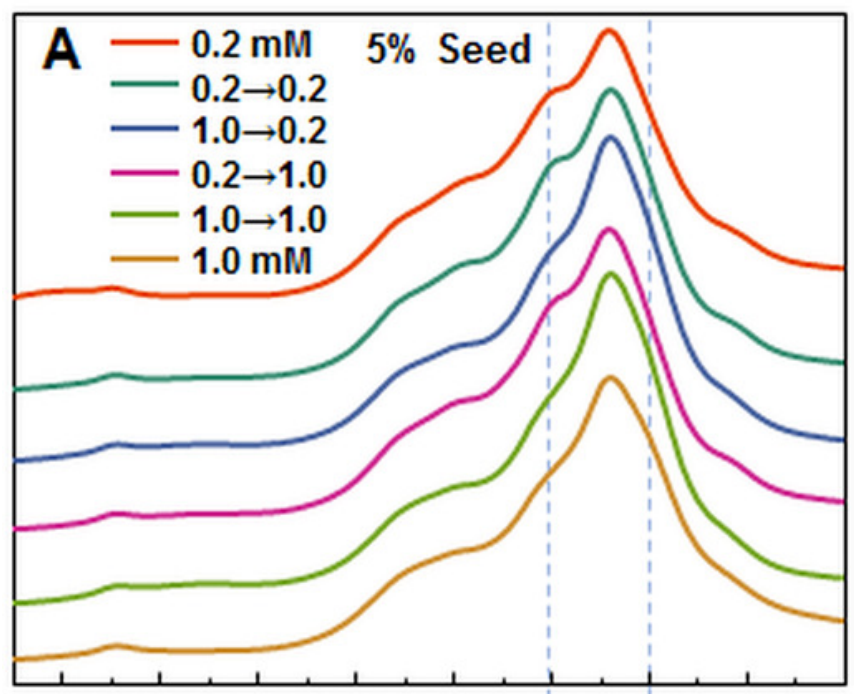

174017201700168016601640162016001580

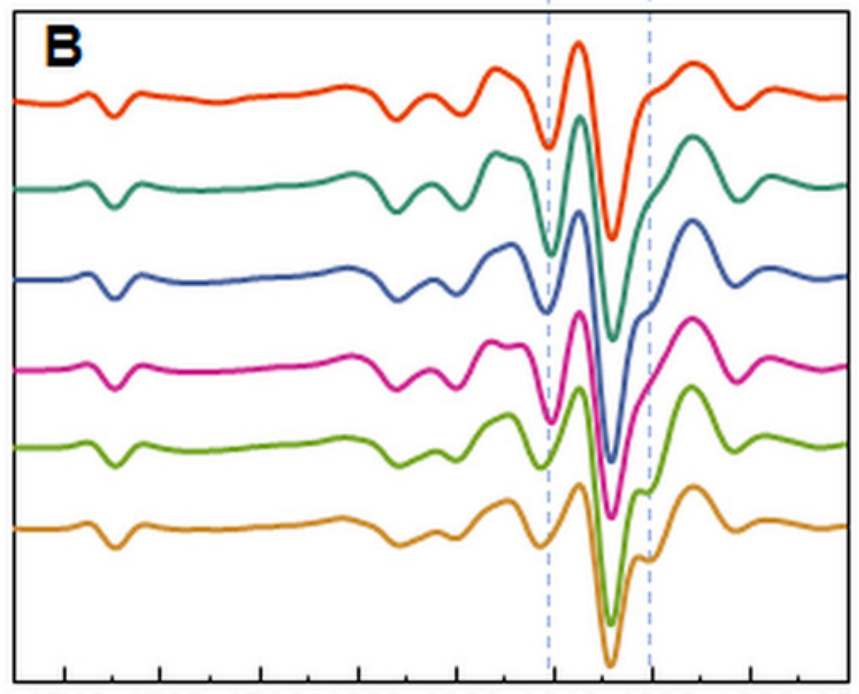

174017201700168016601640162016001580 Wavenumber $\left(\mathrm{cm}^{-1}\right)$

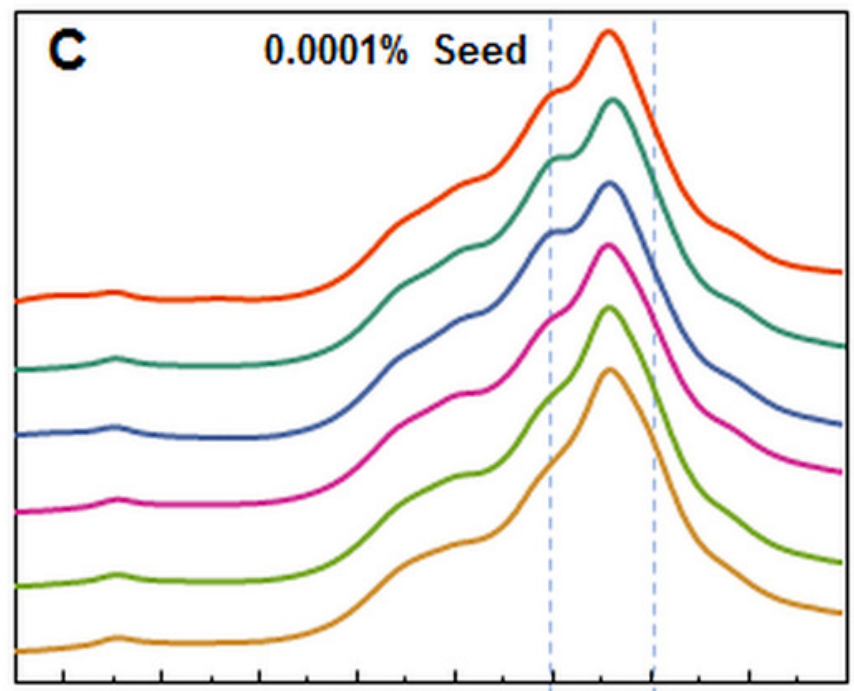

174017201700168016601640162016001580

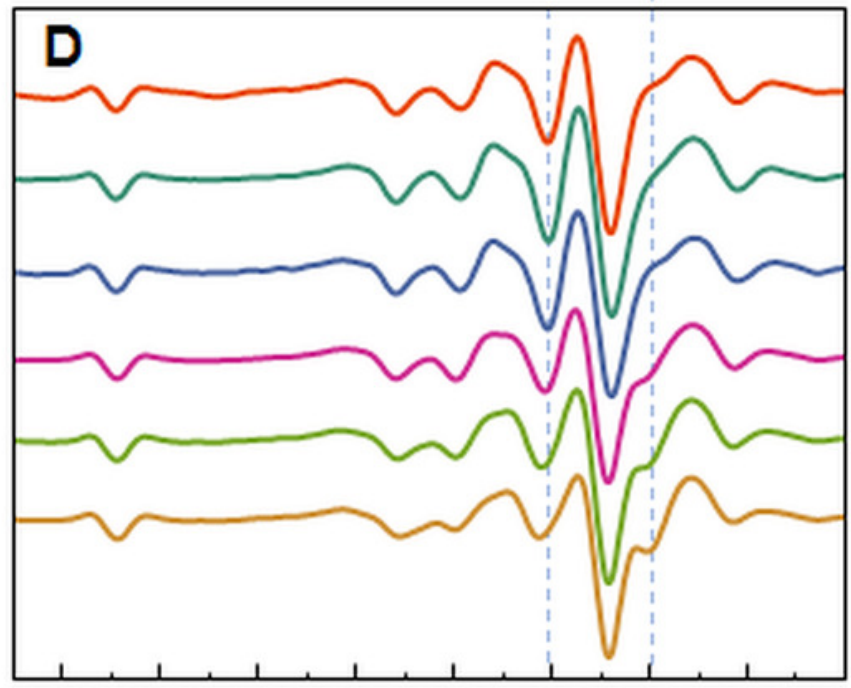

174017201700168016601640162016001580 Wavenumber $\left(\mathrm{cm}^{-1}\right)$ 


\section{Figure 5}

AFM analysis of seeded insulin fibrils.

Insulin fibrils resulting from seeding $0.2 \mathrm{mM}$ insulin with $\mathrm{LCF}(\mathrm{A}, \mathrm{E}), 0.2 \mathrm{mM}$ with $\mathrm{HCF}(\mathrm{B}, \mathrm{F})$, $1.0 \mathrm{mM}$ with $\operatorname{LCF}(\mathrm{C}, \mathrm{G}), 1.0 \mathrm{mM}$ with HCF ( D, H) using $5 \%$ or $10^{-4} \%$ of preformed fibrils respectively. Insulin fibril height distribution with box plots indicating the interquartile range and errors bars are for 1 standard deviation. Sample size for each ANOVA test was 50. 
Height (nm)
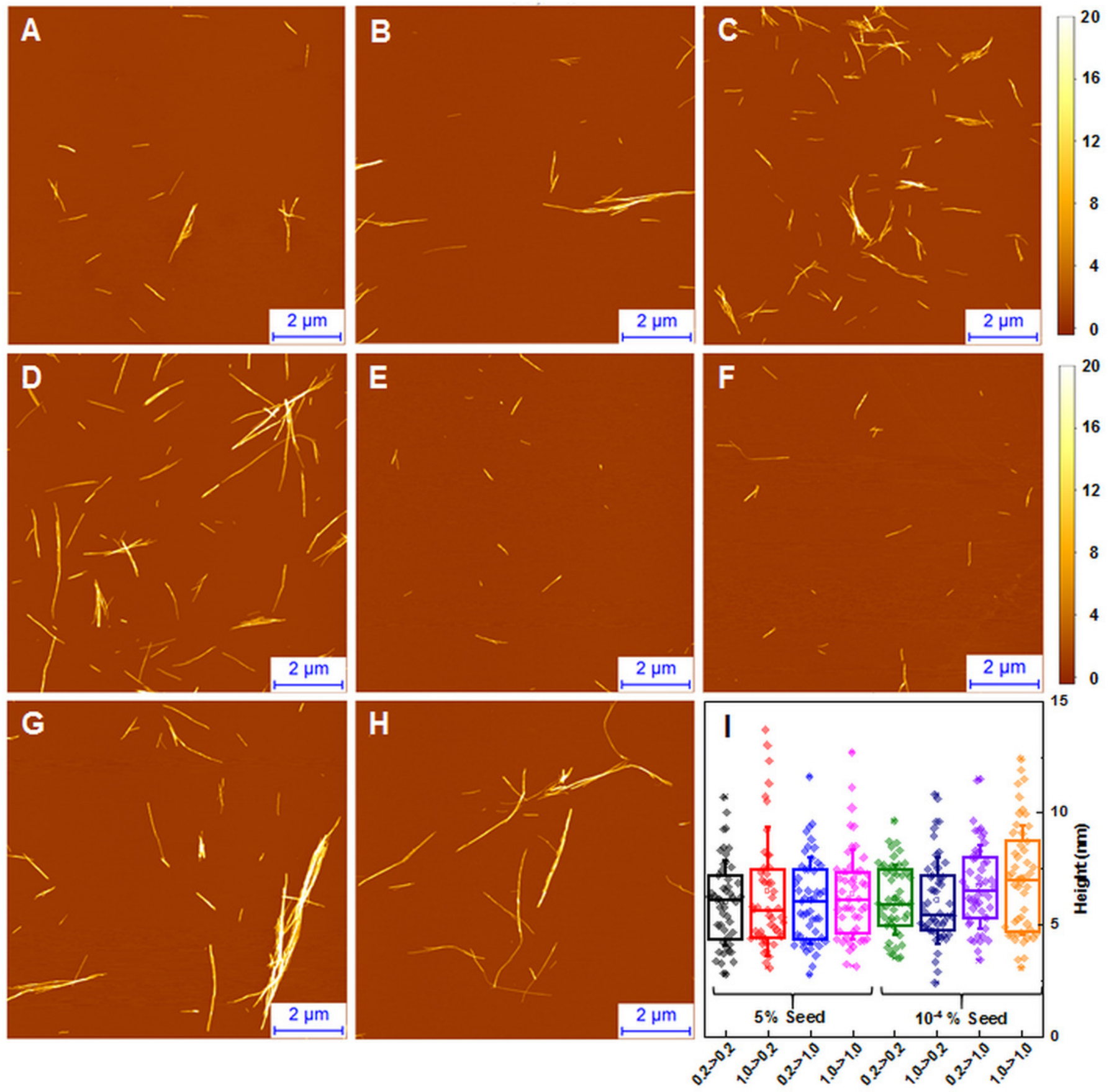